\title{
Verbalizing Sensations: Making Sense of Embodied Sexual Experiences
}

\author{
Myra Bosman $^{1} \cdot$ Rachel Spronk ${ }^{2} \cdot$ Giselinde Kuipers $^{1}$
}

Published online: 3 July 2019

(C) The Author(s) 2019

\begin{abstract}
Embodied experiences are strongly felt, but hard to communicate or verbalize. This article examines people's articulation and sense-making of their sexual sensations, during interviews, to gain insight into what we refer to as a "perceptual loop" between embodied sensations, body-sensorial knowledge, and social meanings. We employ a combination of interactionist and practice theory to study sex as an embodied practice that unfolds in interactions. We analyze this using in-depth interviews with 34 Dutch women and men about their sexual interactions. We focus on how they make sense, with their partner and with the interviewer, of their embodied experiences within an interview setting that requires them to verbalize their experience. Participants communicated these embodied sensations by (1) using multiinterpretable sensory wordings; (2) drawing comparisons between different sexual sensations; and (3) referring to other sensory sensations, and by exemplifying their effect (e.g. breathing, shrieking). We argue that to analyze people's verbalized understanding of their sensations, we need to attend to an ongoing feedback process: sex both shapes and is shaped by social meaning and cultural knowledge. Embodied sensations are not the material onto which the social is mapped. Instead, embodied practices and interactions mediate and shape the social, leading to body-sensorial knowledge. While sex, as an intense embodied practice, is a strategic case to show this process, this "perceptual loop" between embodied practice and bodysensorial knowledge occurs in all human (inter)actions.
\end{abstract}

Keywords Sex $\cdot$ Sensations $\cdot$ Joint sense-making $\cdot$ Interviews $\cdot$ Body-sensorial knowledge

Embodied experiences are strongly felt, but hard to communicate or verbalize. While these experiences can tell us much about human life, researching them presents us with practical

Myra Bosman

M.Bosman@uva.nl

1 Department of Sociology, University of Amsterdam, Nieuwe Achtergracht 166, 1018

WV Amsterdam, The Netherlands

2 Department of Anthropology, University of Amsterdam, Nieuwe Achtergracht 166, 1018

WV Amsterdam, The Netherlands 
and, sometimes, ethical challenges. This is particularly evident in the study of sex. Because of ethical and practical barriers to studying sex directly, social scientists mainly rely on oral and written accounts of people's sexual practices and experiences, like questionnaires and interviews. While speech is an important medium for articulating and reflecting on experiences, it does not entirely capture the "humping and pumping, sweatiness or sexiness" of sexual interactions (Plummer 2003, 525). Contemporary sex research tends to not systematically address the complexities inherent in verbalizing embodied experiences and sensations, be they sexual or otherwise. Instead, sex research often takes informants' verbal accounts for granted, using them as an end product ready for qualitative and quantitative analysis. In this article, we turn our attention to the process of verbalizing embodied sensations itself. We ask: How do interviewees identify and communicate their sexual experiences, and what sort of knowledge is produced in this process? We show how our 34 informants capture their embodied sensations in speech, and how, through this process, social meaning is created. Ultimately, we investigate how embodied sensations relate to social meanings.

In our study on heterosexual sex in the Netherlands, we found that reporting on embodied experiences can be challenging for informants. These experiences were however crucial to goal of the study: understanding how and when heteronormativity informs heterosexual sexual practices and encounters. Our interviewees frequently expressed difficulties in putting their sensations into words. As informant Patrick explained: "I know exactly how it feels but (...) it is quite hard to describe it." Patrick referred to the tacit knowledge that springs from first-hand experience yet cannot be communicated because of the limitations of language. Although it can be said that experiences in general are hard to verbalize (DeVault 1999, 68-69), sexual sensations are particularly challenging to articulate. They therefore provide an interesting case to further investigate how embodied experiences relate to processes of meaning-making, and how such processes are embedded into wider socio-cultural meaning systems. We are especially interested in those moments that interviewees find "language wanting" for describing their experiences (DeVault 1999, 69), and subsequently show how they still manage to capture their embodied sensations in words.

We investigate how people make sense of embodied sensations by analyzing the various ways our informants understood and communicated these experiences in an interview setting. As research on sexual experiences strongly depends on verbal articulation, sex is a strategic case to study this process. Firstly, sexual experiences are embodied, yet not consistently researched as such (Jackson and Scott 2001, 2007; Milani 2017). Secondly, studies of embodied experiences tend to focus on the uncommon-boxing (Wacquant 2004), religious ecstasy (Csordas 1997) — or the unpleasant, like pain and suffering (Wilkinson 2001), bypassing mundane, positive, and more widely shared embodied experiences such as having sex (by oneself or with others). Thirdly, privately experienced sexual moments occur within a wider context in which dominant ideas on what sex should be - and feel-like are widespread, specifically concerning heterosexual sex (Jackson and Scott 2001, 2007).

By zooming in on the process of joint sense-making of embodied experiences during the interviews, both between the partners and between the partner(s) and the interviewer, we investigate how an embodied, mostly non-verbal practice like sex both mediates and shapes the social world. We see this as an important addition to contemporary sex research, which often employs discursive analysis and hence sees embodiment as the product of discourse (Spronk 2014). However, as Spronk $(2014,7)$ writes: "The body is not an empty vessel that can be filled by social meanings of sex: it is needed to bring those meanings into the world." We therefore turn our attention to the ways that embodied interactions produce the social. The process of verbalizing 
sensations in the course of an interview highlights how embodied practices and cultural knowhow are connected in a "perceptual loop," an ongoing feedback process between social meanings and embodied sensations that results in "body-sensorial knowledge" (Spronk 2014). This knowledge is the product of sexual doing, thinking, and feeling; but it also informs the doing, thinking and feeling of sex, in concrete, embodied social interactions.

The Netherlands is a strategic site to study sexual practices, and especially the verbalization of sexual sensations. Dutch sexual morality is generally understood to be exceptionally liberal, yet it is regulated by the normative premise that "sex and love should be combined" (Hekma 2005, 212). Consequently, sex is normalized, even among teenagers, provided that it occurs within a loving encounter, preferably a stable relationship (Schalet 2011). Moreover, the Netherlands is characterized by low levels of religiosity, which fosters relatively liberal attitudes towards sex, and public rejection of religious interference with sex and sexuality (Mepschen et al. 2010). The normalization of talk on sex and sexuality in Dutch public debates is exemplified by its regular appearance in popular and written media, from teen magazines to newspapers, and the many (primetime) TV programs that explicitly address issues on sex, targeting both young and adult audiences. Additionally, in 2012 the Dutch government implemented mandatory sex education in primary and secondary schools to promote sexual health and sexual diversity.

Yet, we found that even people who grew up and live in a society where sexual topics are "openly" discussed, still struggle to articulate their embodied experiences. This suggests that difficulty in speaking about sex is not related to its supposed taboo character. Instead, we argue that speaking about sex is difficult because sex revolves around embodied interactions and practices, and thus draws upon knowledge that is rarely put into words.

\section{Studying Sex as Practice, Interaction, Meaning-Making}

Research that includes both observations and verbal accounts of sexual practices (e.g. Kinsey et al. 1948, Kinsey and Pomeroy 1953), has to a large extent been replaced by questionnaires and interviews on sexual activities and attitudes within the field of gender and sexuality research (e.g. Laumann et al. 1994) and, to a lesser extent, embodied sensations (Frith 2013; Hite 1976; Jackson and Scott 2001, 2007, 2010). As a result, language has become the main medium for studying an essentially physical experience. This has several consequences for the field of gender and sexuality research. First, the study of sex increasingly focuses on verbal, post-hoc accounts of individual sexual experiences and - especially-individual attitudes vis-à-vis sex. Second, a shift occurs where the study of sex becomes the investigation of sexuality: the larger conglomeration of ideologies, moralities, and discourses that are socially constituted, historically grounded, and dependent on cultural contexts (Foucault 1978; Jackson 2006; Rubin 1984). As a result of these individual and discursive perspectives, erotic interactions, practices, and imaginations receive less attention in the sociology of sex. Moreover, the sociology of sex suffers from the larger problem with social theory: the lack of attention to emotions and embodied sensations as forms of knowledge and drivers of human behavior (cf. Crossley 2005; Csordas 1990; Stets and Turner 2014; Turner 2009; Wacquant 2004).

In response, scholars have proposed to redirect attention to the everyday practice of sex, and to focus on the interaction between sexual partners (Jackson and Scott 2010, 2017; Plummer 2003). We draw here particularly on the works of Jackson and Scott (2004, 2007, 2010, 2017), who put forward the relevance of interactionism and practice theory for the study of sex. As 
they argue, an interactionist approach takes the "the everyday gendered doing of sexuality in interaction" as a central point of analysis, thereby locating sexuality "within wider patterns of sociality" (Jackson and Scott 2010, 812). This approach views sexual conduct as entailing "actively 'doing sex,' not only in terms of sexual acts, but as making and modifying sexual meaning" (Jackson and Scott 2010, 821; cf. Spronk 2014). However, since interactionism traditionally pays little attention to embodiment, Jackson and Scott integrate this interactionist approach with practice theory, in order to conceptualize sex as "integrative performance," consisting of "active agency" but also "predictable, even routine, patterns" that are expressed through embodied actions (Jackson and Scott 2017, 70; cf. Warde 2016). This combination of interactionism and practice theory allows scholars to understand sexual interaction as embodied practice through which social meaning is produced.

Jackson and Scott $(2001,2007)$ thus provide a most helpful conceptualization of the relation between the social and embodied sensations. They identify "objectified, sensory and sensate embodiment" (Jackson and Scott 2007, 99; cf. Lindemann 1997) as distinct but interrelated processes that are reflexively understood by the self:

First, the body can be an object of desire or of another's sexual acts; second, the embodied self is capable of sensual awareness of another - this is a self who sees, smells, touches; third, the embodied self also has the capacity to feel the emotions and sensations associated with erotic desire and sensual pleasure (Jackson and Scott 2007, 98).

Central to Jackson and Scott's analysis are processes of reflexivity, which enable "embodied decoding of sensation" and "internal states" as "sexually significant" (Jackson and Scott 2007, 100). The self is understood here as a reflexive actor, capable of experiencing sensations, and interpreting and making sense of what is experienced. Studying this process of sense-making provides us with crucial information about how the social is constructed; how sensate and sensory experiences are jointly formed by and inform our understanding and experience of sex. For instance, how sexual pleasure is understood as located in certain body parts, or what sexual sensations are deemed acceptable, and in turn, how these understandings affect our embodied sensations (Jackson and Scott 2001, 102). Sensate and sensory embodiment are a crucial part of body-sensorial knowledge, but, as our interview data show, the embodied self cannot always articulate what is experienced. This is not because these sensations are pre-social or pre-discursive, but because people experience these sensations as beyond, or outside the realm of, language. This is not necessarily the case for all embodied sensations. Orgasmic sensations, for instance, are often captured in language, discourse, and images present in popular culture (Bordo 1999; Frith 2013; Jackson and Scott 2007). Such codified forms enable people to verbalize their own embodied experiences. Other embodied sensations, however, are much harder to verbalize. Yet it is precisely for this reason that they provide essential information to understand not only people's sexual experiences, but also the relation between the body and the social.

This study builds on Jackson and Scott's work, but focuses on joint sense-making of sexual encounters. This process of sense-making happens, first, in the sexual act itself and, secondly, in interaction with other media (be it persons, objects, phenomena). In other words, our interviews highlight two different forms of sense-making related to sex. We take the interactions-cumpractice theory approach developed by Jackson and Scott as point of departure for analyzing both. Practice theory helps to understand sex as a practice that can be learned and reflected on. Through reflection and learning, people create and negotiate social understandings of what embodied sensations are considered part of sex, and come to identify sensations as pleasurable and exciting (Jackson and Scott 2017, 72). Practice theory also aids in examining the routine and 
habitual actions that are part of sex, and which to a large extent occur "in a state of distraction" (Warde 2014,292). There are multiple pathways by which practices become routine. In the case of sexual practices, embodiment plays an essential role. Like Jackson and Scott $(2017,74)$ we hold that sex, much like eating and cooking, is informed by "taken for granted embodied knowledge" (our italics) and "know-how." Additionally, much of what happens during sexual encounters is informed by feeling or wanting to feel certain embodied sensations. Thus, the body is not only an instrument through which sexuality is expressed, it also is the mediator of practical cultural knowledge, the driver of individual motivations, and the means by which all this is communicated to others. How these embodied sensations inform sexual interactions, and thus create social meaning, is a central focus in our research.

The interactionist approach, on the other hand, leads us to examine how partners jointly negotiate and make sense of embodied sensations and sexual practices more generally. It holds that subjects are continuously negotiating normative expectations and assumptions in the coproduction of an interaction, thus creating and recreating meanings and, ultimately, the social (Fine 2001; Zussman 2004). In this study, we are interested in how people negotiate the interplay between normative ideas about what sex is, what it should feel and be like, and sexual practices as they take shape in embodied interactions. The combination of practice theory and interactionism allows us to focus on the "various layers of experience" (Berger and Luckmann 1991, 35) that are part of sexual interaction. In combination, these approaches provide insight into how processes of meaning-making unfold in interaction, as people strive and sometimes struggle to put into words sensations and practices that are more typically bodily experienced than verbally expressed.

It remains a challenge to fully integrate this social interactionist/practice approach with the dominant discursive approach to the study of sex. According to Reckwitz (2012), we are hampered here by the legacy of classic social theory where feelings - both in the sense of emotions and of embodied sensations - are not considered part of the social, but as the substance onto which the social is mapped. Consequently, both emotions and the body are mostly considered products of the social. Yet, Reckwitz argues $(2012,249)$ "every social practice involves an affective-perceptive structuration worth of analysis." Interviews require people to verbalize, and thus to reflect on their embodied sensations, and it is through reflection that the experience acquires a new layer of meaning (Schutz in Wagner 1970, 63). In order to bridge discursive and interactionist approaches, or words and sensations, we rely on Spronk's notion of a "perceptual loop": "A reinforcing process between social knowledge and bodysensorial knowledge, between sexual practices as mediators and shapers of social knowledge. 'Doing' sexuality is therefore the productive moment; in the experience of sex(uality), social meanings and sensations converge and are mutually constitutive" (Spronk 2014, 7).

This notion of body-sensorial knowledge points to the mutually constitutive process of doing, thinking, and feeling in the creation and negotiation of meaning. Various layers of experience cannot be investigated as a successive process, where reflection follows practice, or the other way around. Nor can they be taken as the result of discourse where practices are mediators of regimes of power. Instead, the perceptual loop foregrounds practices as mediators and shapers that happen in the course of concrete interactions. We call this a perceptual loop to highlight the process of understanding the social, using the senses (Merleau-Ponty 1962). Emotions and feelings are therefore not the material onto which the social is mapped. Instead, they are the modalities for shaping the social.

Sense-making, which we understand here as joint, reflexive interpretation embedded in interaction, is an important aspect of processes of meaning-making. While our interview data 
seem to privilege the personal level of experiences, they are the result of ongoing processes of doing, thinking, and feeling that are socially (in)formed. Our interviews therefore not only shed light on the relation between embodied experience and cultural knowledge during sex. They also bring out the process by which such experiences become intersubjective knowledge in the course of an interview. During interviews, too, people make sense of their personal experiences through the perceptual loop between practice, cultural (but often tacit) knowledge, and interaction.

Like a sexual encounter, the interview situation brings out the mutually constitutive process between social meanings and practices. Interviews facilitate a joint understanding of the experiences during the interaction between researcher and informant(s) (Bjørnholt and Farstad 2014), who are both actively involved in "meaning-making work" (Holstein and Gubrium 1995). In the interviews on which this article is based, people were invited to reconstruct the process of doing, feeling, and thinking, guided not only by the interviewer, but also by their partner during couple interviews.

Informants had to verbalize sensations that were difficult to put into words in the first place. Hence various forms of probing were used during the interviews, which were specifically designed to trigger this bodily knowledge, such as the use of a Body Map (see method section below). The verbalization of sensations, then, becomes the data to investigate the perceptual loop between the body and the social, and thus, the relation between sensory experiences and social meanings. To do so, we developed a research design that enabled us to investigate the process of doing, feeling, and thinking as much as possible, that is, being shaped and limited by the challenges of verbalizing sensations. Following the theoretical perspective outlined here, we see these layered interactions and constructions of sexual not as different ways of getting at an underlying something that is "real," but as various moments in a sense-making process (see Fig. 1 in appendix).

\section{Methods and Data}

This article is based on an interview study among 17 Dutch man-woman couples ${ }^{1}$ about their sexual experiences, to examine how partners understand their sexual interactions and negotiate norms on (hetero)sexual sex and sexual pleasure. The in-depth semi-structured interview series consisted of two couple interviews followed by one individual interview. All were conducted by the first author.

The joint interviews touched upon various themes, including the couple's relationship, how partners think about social norms, the sexual interactions between partners, and their embodied sensations. Regarding the latter, informants, for example, did a Touch 'n' Feel assignment: Partner A described step by step how s/he touched Partner B during a (self-chosen) previous sexual encounter, and Partner B explained what sensations were felt. Afterwards, the partners reversed roles. The individual interview dealt with the informant's social background and sexual biography, and then turned more profoundly to the embodied sensations that are felt during self-sex and sexual interactions in general (not necessarily with the current partner). A Body Map, an A4-size drawing of the outlines of a gender-neutral human body, was given at the end of the second joint couple interview as "homework" (see Figure 1 in the appendix).

\footnotetext{
${ }^{1}$ The term man-woman partners refers to couples in which one partner identifies as a man and the other partner identifies as a woman. The term is chosen instead of heterosexual partners or couples, to avoid the misunderstanding that partners should identify as heterosexual.
} 
Partners were asked to indicate with pen or (colored) pencil (1) where they like to be touched or touch themselves sexually and, (2) where they feel the effects of this in their body. Both the Touch 'n' Feel assignment and the Body Map were designed to aid informants in reflecting on their embodied sensations (for more information on the use of Body Maps, see De Jager et al. 2016, MacGregor 2009). The overview of an actual body or a drawn body made the exercises less abstract, which facilitated the sense-making process. Additionally, informants resorted less to metaphorical speech to communicate what they experience.

Thirty-four women and men (i.e. 17 man-woman couples) participated in all four interviews, resulting in 68 interviews. The informants were between the ages of 25 and 55, with a mean age of 34.2 (31.9 for women and 36.5 for men). Nearly half of the interviewees identified as Christian (14 out of 34) and grew up in Christian families. Most informants had completed higher education ( 30 out of 34 held a college or university degree) and were white, and all of them were brought up in the Netherlands. Several informants had parents with mixed (mainly European) nationalities, and one informant had an ethnic minority background. All informants indicated that they were in a romantic relationship with their partner, and had been together between three months and 20 years. No relation was found between the duration of the relationship and how partners talked about sex during the interviews, nor between the articulation of embodied sensations during the joint and individual interviews. This could be explained by self-selection in the sample: those couples who signed up for participation were likely more willing and used to talking about sex with each other. The data presented here were taken from both individual and joint interviews. We found no difference between individual or joint interviews in explicitness or detail concerning the verbalization of embodied sensations (see also informants' quotations in subsequent sections). This is not to dispute that both individual and joint couple interviews come with their own advantages and challenges (Allen 1980; Duncombe and Marsden 1996; Heaphy and Einarsdottir 2012), but these do not seem to have impeded our informants from articulating their embodied sensations.

In order to facilitate the informant's sharing of sexual experiences and sensations, both with the interviewer and with each other, the interviewer invested much time in establishing rapport by creating an atmosphere of trust and openness. Although most couples were very open with each other about sex, they had never participated in a research project on sex before. To make partners feel comfortable, several measures were taken. First, conducting multiple interviews proved highly productive in establishing a bond of trust between the interviewer and informants. Also, before and after the interviews, which in all but two instances took place at the interviewees' homes, there was everyday talk about hobbies, holidays, and family matters. Sometimes the interviewer was invited for lunch or dinner, or got picked up from the station, which provided other opportunities for casual conversations. Second, the partners were well informed about the content and explicitness of the interviews before the start of the interview series. The interviewer had a phone call with each informant (sometimes together) to explain the research aim, give examples of interview questions, and to answer any questions before the interview series. Third, the interview format allowed partners to get used to being interviewed together, and started off with general questions about how they spend their time together and apart, how they met and what they like about each other. Gradually, the questions steered towards sexual practices and sensations. The interviewer adapted to the couple's language use by copying the wordings informants themselves used (e.g. intercourse instead of fucking, or the other way around). Importantly, the interviewer was respectful but did not avoid being explicit in her questions, which several informants seemed to appreciate and helped them overcome their own initial embarrassment (see also below). In conjunction, the partners' open attitude, their curiosity about each other's stories, the interviewer's experience interviewing about sex, the in-depth interview questions, and 
a mutual interest in the topic of sex created constructive circumstances for partners to talk, in front of each other and the interviewer, about their sexual experiences and embodied sensations.

Informants were gathered through the first author's social and professional network, interviews with different media, snowballing, social media, newsletters, newspaper and online advertisements, and through flyers in public spaces (e.g. bars, sex shops, health practitioners' waiting rooms). The research project's website and Facebook page enabled easy sharing of information on the study and provided elaborate material on the project itself, as well as participation in the interviews. All informants received one or more pseudonyms, with multiple pseudonyms used if requested by the informant to also present data from the individual interviews anonymously. The procedures for safeguarding confidentiality and consent have been approved by the Ethics Committee of our home university. The first author conducted and fully transcribed the interviews, thereby also noting instances of laughter, silences, any other sounds, and movements of informants. While interviewing, transcribing and (re)reading the interviews, it became apparent that informants often spontaneously mentioned the challenges of verbalizing their embodied experiences, which instigated the writing of this article. The data were then fully coded and categorized using Atlas.ti to examine this pattern more thoroughly and systematically.

\section{Talking about Sex: “Talking about Things You Haven't Talked about before"}

Before turning to how informants make sense of their embodied sensations, we briefly touch upon the particularity of interviewing about sex. In the case of sexual experiences, it should be considered that informants may feel uncomfortable disclosing their intimate moments (Hockey et al. 2007, 98-99; Van Hooff 2013, 93). Despite a possible self-selection in the interview sample (i.e. couples willing and wanting to talk about sex), feelings of shame or uneasiness occasionally hampered the process of sense-making during the interviews, indicated, for instance, by laughter and silence. Occasionally, naming specific sexual acts or body parts was accompanied by hesitation. Tina, for example, burst into laughter when she used the word "cock" during the Touch 'n' Feel questions, because "it sounds so stupid to say."

Interviewees sometimes referred to their uneasiness in terms of a "threshold" (David) or a "mountain" (Eric) that had to be overcome, because they were now "talking about things you haven't talked about before" (ibid). Or as Eric noted: "Normally you would need alcohol to tell this to someone." Thus, interviewees felt that a boundary had to be crossed in order to share their privately experienced sensations. This is related to the notion that sexual experiences are regarded as personal and private in Dutch society, as in many other societies, and people do not regularly disclose these to others (Bosman 2012; Schalet 2011). Consequently, some informants had not yet established a habit of openly thinking and talking about their experiences, for instance because "no one has ever asked about it" (Richard), which meant some informants "had to get used to it" (Victor) during the interviews. Yet, few informants indicated feelings of discomfort, while others very much welcomed the open and explicit questions about their embodied sensations and provided highly detailed answers in return. Also, it is a topic that some "really enjoy" talking about (Thomas) in conversations with friends or during the interviews.

Some informants showed awareness of their choice of words when describing sexual experiences. In relation to the process of sense-making, this meant that the interviews required 
them to be reflexive of both their sexual experiences, and of how to capture these in "decent" wording (John), as sexual terms are hardly ever neutral in Dutch. Indeed, sexual language specifically comes with its own challenges because there is a "limited vocabulary to talk about sex," which causes "discomfort because it either sounds too medical or too vulgar" (Philip). Through their speech, informants sometimes tried to present themselves in a decent and polite way, which is a more general characteristic of interviews (Pugh 2013). For instance, they used language that they felt more fitting for an interview setting, or occasionally resorted to metaphors. Partners Gina and John, for example, indicated after their first joint couple interview that they were wondering, "How do you talk about [sexual experiences] in a decent way, and, ehm, how do you call it?" (John). Gina added that she was thinking, "Will I call a spade a spade or will I describe it a bit?" John explained afterwards that he initially wanted to say how he likes "to do Gina," but instead phrased his desire for intercourse as "to give her pleasure." This "display work" (Pugh 2013, 50) tells us how informants are consciously and actively using certain terms instead of others, not because it better captures their embodied experiences, but because it better fits the image they wish to convey. After becoming familiar with the interview context, however, this particular group of informants did not struggle so much with avoiding "too medical or too vulgar" talk, but with the limitations of speech itself and with their unfamiliarity with verbalizing their embodied sensations. They tried to find the words that best fit their experiences. This set in motion the reflexive process that we describe here as a crucial part of the perceptual loop between embodied sensation and social meaning.

\section{Identifying Sensations: "What Do I Feel?"}

Sexual embodied sensations comprise a broad spectrum of feelings, from experiences of arousal to orgasm, being touched, stroked, licked, clutched, having intercourse, and so on. The interviews not only required informants to talk openly about their sexual experiences, but also prompted them to be reflective about what they feel, how they experience this, and in what situations. Through talking about their experiences and sensations, people "form reflexive thoughts and equally (...) become aware of those thoughts" (Crossley 2001, 112). Still, identifying what is felt proved to be challenging at times. This is related to the informants' strong desire to fully experience sexual pleasures (sometimes mixed with other sensations), while they also considered sex to be a moment at which they either are not consciously aware of what they feel or prefer not to be. During the "heat of the moment," they want to let go. This apparent contradiction is explained by distinguishing between experiencing sensations and being aware of what is experienced. It is a distinction similar to what Weber (1978, 21-22 in Warde 2016) puts forward in his discussion of habitual behavior - a central notion in practice theory. Habitual, or rather "deeply engrained," behavior often happens "in a state of halfconsciousness or actual unconsciousness [Unbewusstheit] of subjective meaning. The actor is more likely to 'be aware' of it in a vague sense than he is to 'know' what he is doing or be explicitly self-conscious about it” (Ibid.). As Helen summarized:

It's not something I'm very much thinking about during sex. And I really like that I'm not so consciously focused on these [feelings], when I can let go of being consciously aware.

This lack of awareness made it challenging to identify sensations. Moreover, informants reported an unwillingness to be consciously aware of what one experiences at that moment. This idea of not being aware of what is felt is further elaborated on by informants who 
understand their sexual sensations as out of their "control" and "just happen[ing] to you" (David) or who wish to "not think too much" about them while having sex, because "it could be distracting" (Steven). Kevin related his inability to be aware of what is happening to the "state of trance" he experiences during sex, in which he "just did things in a kind of state of arousal" and "maybe was a bit of a different person." This left Kevin to wonder: "What do I feel, or what did I feel?" These responses show how sex is perceived as a partly habitual, halfautomatic experience; a "praxis" that unfolds without conscious reflection. Simultaneously, interviewees described their experiences as one in which their body "takes over," resulting in a flow of actions and reactions that are not necessarily habitual, but happen in a state of lessened self-consciousness. This observation has also been made about other forms of intense embodiment, like religious ecstasy (Csordas 1997) or boxing (Wacquant 2004). Wacquant (2004, 97) notes that boxers appear to have "a contradictory view of the 'mental' aspect of their activity," as they argue that boxing is both about "thinking" and "no ratiocinating." While boxers need to be in control of their body and emotions (Wacquant 2004, 93), they also rely on a learned "comprehension of the body," when boxing moves are "inscribed within one's bodily schema" after repetitive practice (Wacquant 2004, 69). Even when close to a knock-out, the experienced boxer's body "continues to box on its own, as it were, until they regain their senses" (Wacquant 2004, 96). This points to a similar mixture of habit and flow, simultaneously with and without conscious awareness, that our participants encounter during their sexual practices.

Importantly, interviewees tended to make sense of their embodied sensations as prediscursive, and hence possibly a-cultural, viewing it as a purely bodily experience disconnected from a wider social context. In this understanding, sexual experiences are characterized by a certain degree of unawareness. During the interviews, however, interviewees were asked to articulate their embodied sensations and thus "undo" the unawareness that is part of the sexual experience. The difficulty in verbalizing sensations, as mentioned by the participants, is then not only due to the limits of language, or because of the complexity of what is felt, but also because it may change the experience itself.

Thomas gave a detailed account of his reluctance to "analyze" his embodied sensations, and how he found it "really difficult to think about it".

Interviewer: What makes it so difficult?

Thomas: I think I'm not so consciously aware of it and I don't want to be so conscious about it (...) 'cause I just want to experience it (...). That's just what I'm like, and I've got that with all kinds of things. And sometimes the pureness of something is lost (...) 'cause you consciously analyze something that you previously just experienced. And the same goes for this [Touch 'n' Feel assignment], uh, I noticed I was thinking about it and that I think like, yeah, I actually don't want to analyze why-I'm just being touched, and I just like it.

Interviewer: Yes.

Thomas: So, it's just really weird to think about it, but I do like [the assignment] 'cause I'm always up for an experiment. (...). But then I noticed that I really had a lot of difficulty doing it. And just now, I was looking at that drawing [i.e. Body Map], and I thought: "Yeah, I could color the whole little figure [i.e. body] red, and, eh, that's how it really feels." 
Thomas related the difficulty he encountered in identifying his embodied sensations to concerns about the possibility of corrupting his sexual experiences through analyses (at that moment, and in hindsight during the interviews). The act of analyzing may cause an irrevocable loss of "pureness" of the experience, and thus spoil future sexual encounters. Additionally, Thomas signaled that his embodied sensations were hard to disentangle: the entire body was understood as sensory and sensate, hence the entire Body Map could be colored "red." Similarly, several participants pointed out that it can be hard to locate and isolate embodied sensations, because they all feel to be so intertwined. Thomas made one final interesting point. He added that studying interviewees' embodied sensations could be useful when people have "certain traumas" and "don't want to be touched somewhere," implying that his own "ordinary" feelings do not need the same attention; that would be "weird." It shows how routine and habitual Thomas's sexual experiences feel to him, and so they are not in need of an explanation other than that he "just like[s] it."

These cases also show how informants understand their embodied sensations as being felt without consciously monitoring them. It is viewed as "embodied knowledge" and not as "discursive knowledge" (Crossley 2001, 102). Although body-sensorial knowledge is crucial to understanding and meaning-making in sexual encounters, it is also, as the above examples show, difficult to access this knowledge through interviews. These reflections and accounts show the specificity of such body-sensorial knowledge. Speech is then taken as a medium, or actor, that facilitates processes of reflection. This is the first step in enabling us to understand how body-sensorial knowledge relates to social meaning.

An important way through which informants reflected on and made sense of their embodied experiences, was by comparing and contrasting the effects of different ways of being stimulated. Making comparisons was a way for informants to identify their sensations, and aided in communicating that there are differences between these sensations. The one is more intense, pleasurable, or satisfying than the other. As in the case of Sophie, who, during the second joint interview, described how her partner Thomas stimulates her:

(...) then Thomas is licking the inside of my ear and doing-and that is just as intense as my pussy being licked, you know. But maybe even more intense, because he then often simultaneously does something vaginally. Oh, that is really such a sensation. But the other day, he really totally [grinning] licked it off. Really super good.

Sophie reflected on how different ways of being stimulated intensify her pleasure, which indicates how she understands these experiences in contrast with each other. This is also apparent in her partner Thomas's account who described, during the same interview, how he prefers variation in the stimulation of his penis and testicles. He gave a detailed account of how "a blow job is of course more a sucking stimulus", which after a while becomes "more ordinary" (i.e. a feeling he gets used to). Because, as Thomas explained:

If you make the same sucking movement over and over, it feels less pleasant. But if you then squeeze my balls, or lick me like a lollypop, and then suck me again—yes [sighs]. It's the variation that makes it really pleasant.

Thomas's reflection exemplifies how informants base their preference for sexual stimuli on their already existing body-sensorial knowledge. This shows how the process of sense-making is related to previous experiences. Both examples show that the embodied "fleshy knowledge and memory" is based on routine and habits (Allen-Collinson 2009), which in turn is informed by the sexual sensations themselves, and shapes the sexual interactions. Interestingly, Thomas 
criticized dominant views of fellatio that do not go beyond certain simplistic accounts (apparently). This demonstrates how the practice of sex(uality) creates new knowledge and meaning-for individuals and for couples - which is then acted upon (Fine 2001; Spronk 2014). These examples indicate the importance of a particular kind of knowledge that is rooted in the body and in the senses, which we refer to here as body-sensorial knowledge. Identifying sensations (the bodily experience of identification) both during sex, and during reflections on sex, triggers the perceptual loop of reflecting on and comprehending one's experiences and, hence, (re)creating the social world.

This perceptual loop is also apparent in instances in which people know how a certain stimulation should feel based on previous experiences. This shows how body-sensorial knowledge stems from and is based on earlier sexual experiences and interactions. When the "right" feeling is absent, and uncomfortable or painful sensations are felt, participants explain that there is a mismatch between their expected and actual embodied sensations. In these cases, participants tend to not only describe the sensations that are felt, but also identify the ones that are missing. At those moments, the half-awareness associated with pleasurable sex also falters. Casper, for instance, shared how penetrative sex "can feel really sensitive" in an unpleasant way. His partner, Helen, sometimes holds his hips during intercourse, Casper explained, to indicate the "rhythm" and "depth" of thrusting. This is "sometimes annoying" to him because he then cannot "slowly build-up" the encounter. Casper described what he felt:

Interviewer: And sensitive-you mean that you're feeling sensitive? That it [i.e. penetration] feels sensitive to you?

Casper: Yes, it tintelt [tingles] $]^{2}$ in my penis [grinning].

Interviewer: Yes, I see.

Helen: Yes.

Casper: A bit of a kriebelig [tickling] tintelend [tingling] sensation. And then you ejaculate just like that, you know. That it comes out and that you haven't even yet experienced that it feels good or something.

While "good" is quite broad a term to describe pleasurable sensations, this excerpt makes clear that Casper expects these sensations to precede his ejaculation during intercourse. Based on body-sensorial knowledge, in his understanding, and that of many others (Jackson and Scott 2007), ejaculation and sexual pleasure go together in sexual encounters. Here we see how the loop works both ways: the embodied sensation shapes the social understanding of orgasm, which in turn informs the sense-making process of embodied experiences.

Similarly, Jessica described how she used to enjoy it when her partner touched her breasts, but ever since she stopped taking the pill her nipples feel "incredibly sensitive" when she is on her period and so the touching "doesn't feel nice." Jessica drew a comparison between these two situations, when touching her nipples does and does not feel pleasurable. This helped her reflect on her embodied experience of sexual arousal:

$\overline{2}$ The next section explains the use of "tintel" and "kriebel" more elaborately. 
[It's] annoying, because you [Steven] often [touch] my nipples and that, eh, doesn't always feel pleasurable. But when it does feel good then, eh, yes, then I get into the mood. And then it starts to tintel [tickle] a bit. And that's also funny, I always feel that when I get horny that it goes from my belly to my vagina, really that kind of kriebels [tickles]. And then I feel it tintels [tingles] down there.

Like Casper, Jessica explained what pleasurable sensations she expects to experience when certain body parts are stimulated, based on previous experiences. It exemplifies how bodysensorial knowledge is part of the expected and habituated experience of how it usually feels and thus should feel. Also, Jessica, like all interviewees, connected different embodied sensations with each other, and this combination was then made sense of and interpreted as, for instance, arousal (Jessica) or orgasm (Casper). These expectations in turn, lead to certain routines and habitual sexual actions as they become the thread connecting one sexual experience with the others that have gone before, and the future ones that are yet to come.

\section{Communicating Sensations: “I Know Exactly How It Feels, but It's Quite Hard to Describe It"}

So far, we have shown the complexities informants face in reflecting on their embodied sensations and the efforts it takes to, nevertheless, identify and verbalize what they feel. Indeed, informants remarked that it is "difficult to find words for it" (Philip), "hard to put it into words" (Bert), or that you "cannot express it in words" (Tara). The limitations of language they encountered (DeVault 1999, 69; Willig 2007, 223), rendered it "very hard" and "very difficult" to turn their body-sensorial knowledge into speech. These challenges arose not only from the "habituated" or "half-conscious" mood associated with pleasurable sex, but also from a perceived contrast between sensations and language, which the informants viewed as two distinct and unrelated dimensions. Simone explained that this is "because a lot of what I experience is based on feelings" and therefore she did not have "any concrete words in [her] head." Likewise, Philip clarified that it was "difficult" to "translate" a "thoroughly bodily experience" into words, also because he was not "searching for words to capture it (...) during the experience itself." Additionally, several informants indicated that some emotions were untranslatable into words. Whereas the informants found their feelings and the wording to be mutually exclusive, we see this moment of reflection and "translation" as the defining moment of feedback in the perceptual loop between embodied practice and social knowledge.

Despite the individual challenges, the respondents often gave elaborate descriptions of what they experience. Patrick, for instance, provided a rich and detailed example of articulating his embodied sensations. When asked how he felt when he really feels like having sex, Patrick closed his eyes, deeply breathed in and out, and was silent for nearly 10 seconds. He then explained: "I know exactly how it feels but (...) it's quite hard to describe it." Yet, Patrick slowly but surely produced a detailed answer about the "really very strong energy" he experienced in his body, pausing several times for up to 13 seconds, and keeping his eyes closed the entire time:

Patrick: (...) Yes, ehm [12-second silence], what do I feel? Yes, an, eh, warm feeling, often also in my dick, and in my abdomen, kriebels [tickles]. And, ehm, a bit sweaty hands. And being touched feels pleasant. So also when I touch myself, it feels very pleasant but also tense, very yes, pleasantly tense. How do you say that, energetic. 
Interviewer: Intense.

Patrick: Yes, very intense, almost a pounding feeling. Ehm, a wet mouth, lots of saliva ehm [13-second silence]. Well, sometimes also my anus opens, eh, opens a bit.

Interviewer: Yes, yes.

Patrick: [Five-second silence] Yes and everything just gets more sensitive. When you touch me, then it, eh, feels very pleasant.

By taking his time and closing his eyes, Patrick, step by step, made sense of how sexual arousal feels to him. He identified embodied responses (like sweaty hands, anus opening) and embodied sensations (for example, a warm feeling, energetic feelings), showing a high level of reflexivity. This example also illustrates how body-sensorial knowledge is generated in the practice of sex: the experience itself is the basis of knowledge, known by and through the body and formed and informed by social meaning, but also immediately reworked to make it fit the specific sensation. Additionally, it suggests that although informants experienced language as a problem, language may not be the real problem here: it is also the complexity of the embodied experience that is difficult to grasp and make sense of.

To most participants, visualizing their embodied sensations through filling in a Body Map proved to be an effective way of reflecting on and communicating what they feel. Additionally, the accompanying questions (Where do you like to be touched or do you touch yourself sexually? And where do you feel the effects of this in your body?) broke down the steps of identifying, interpreting, and communicating embodied sensations, because they initially focused on the location of the embodied sensations. During the interviews, subsequent questions were asked about the nature of what is felt. By triggering the reflexive process this way, an intermediate step was built into the process of verbalizing embodied sensations. Particularly participants who filled in the Body Map prior to the individual interview (some forgot to do so or lost the Body Map altogether) often put much thought into their embodied sensations. Simone, for instance, used different felt tip markers (red, blue, green, and orange) to color body parts on the Body Map. She differentiated between more and less intense embodied experiences, and additionally, indicated the interaction between various embodied sensations. As Simone explained (see also Figure 1 in the appendix for her Body Map):

Simone: (...) Ehm, I did it from most intense to least intense, so the most sensitive erogenous zones are red (...). My neck, ears, and shoulders, and indeed my back [are red]. And my genitals too, by the way, but that seems kind of self-evident. But, ehm, yes, the green bit, ehm, gets mostly really sensitive if [the red zones] are being touched.

Interviewer: Ah, right.

Simone: So, say Lucas kisses my back and he touches my buttocks, then it's really sensitive. Ehm, even though it's also quite sensitive when he starts with my buttocks, but it gets even more sensitive after he's been there [points at back]. So that's all really connected.

Simone used the Body Map as a tool to capture and communicate her multi-sensorial experience of sex, and importantly, to describe the interplay between stimulation and embodied sensations. We see here how social understandings of sexual pleasure interact with body-sensorial knowledge. The very fact that Simone spoke of "erogenous zones" - a phrase deliberately avoided by 
the interviewer - shows how she understands her body as sexual (Jackson and Scott 2007, 100). This social understanding shaped how Simone made sense of the embodied sensations she experiences. She then used the notion of erogenous zones to verbalize her embodied sensations in a way that was meaningful to her and best captured her experiences. Hence the categorization of more to less "sensitive" body parts, and the descriptions of specific sequences of stimulating body parts through which sexual pleasure is maximized. Like Simone, many participants described their sexual interactions as a build-up of intensifying sexual pleasure, in which a particular sequence of touching and stimulating plays an important role.

During the interview sessions, all informants reflected on their embodied sensations, showing considerable inventiveness in articulating their experiences. One recurring pattern denotes the ways interviewees understand and capture their sensations by using multi-interpretable, but sensorial wordings, in this case kriebel $^{3}$ (tickle, itch/to tickle, to itch) and tintel (tingle, tingling, prickle/to tingle, to prickle). The interviewees used these terms to grasp the mixture of sensations they experience, and their meaning can differ from one informant, interview answer, and context to another. This language use is not coincidental; a particular characteristic of sex is that the same way of touching or being stimulated may feel differently in different contexts and during varying levels of arousal, and this is reflected in the ways kriebel and tintel are applied. In other words, the sensations inform the language that is used to describe and make sense of these very experiences (Cameron and Kulick 2003, 12). Moreover, through reflection and verbalization, social meaning is created. This is an important step in the operation of the perceptual loop.

Kriebel and tintel usually refer to either a way of touching (as verb) or feeling (as noun, adjective, or adverb), but are not related to a specific body part or intention and do not have a specific sexual connotation in everyday Dutch language. Simone, for instance, likes to be kriebelt on her "head (...) while watching TV" and Richard likes to kriebel his anus, which "is not erotic at all" to him. For others, then again, kriebelen is not pleasant when they are not sexually aroused. Depending on "how aroused" she is, Naomi finds it "really unpleasant and kriebelig" when her breasts or the inside of her elbows are touched by her partner. To Victor, the opposite of nice and pleasant feelings is kriebel, unless he is "really aroused, then nothing really matters" anymore. The feeling of tintel, by contrast, is more straightforward in that it usually has a positive connotation and informants used it in relation to sex. When asked to define how tintels or tintelingen feel, generally in relation to sexual arousal or desire, informants elaborated and made sense of their experiences. Sandra, for example, talked about "tintelingen of arousal," Tara explained "a tinteling in your body" like a "feeling of happiness," to Richard "a kind of tinteling" resembles "a kind of feeling of goose bumps," and John described "a tintel" as "a kind of warm feeling throughout your body." The informants thus bended and adapted language to more accurately describe their experiences, and by doing so, created new meanings of these terms and the practices they referred to.

To further tackle the complexities of the embodied experiences and their verbalization, informants also used other creative solutions to verbalize their feelings. Two ways of communicating become apparent as a means of doing so, which exemplify how informants understood their sensations in nonverbal ways and were inventive with language in communicating them. First, informants used comparisons to other sensory experiences to explain what they feel. Feelings of sexual arousal were,

\footnotetext{
3 "Kriebel" or "kriebeling" are nouns, with "kriebels" as its plural. "Kriebelen" is a verb. "Kriebelig" can be used as adjective and adverb.

4 "Tintel" or "tinteling" are nouns, with "tintels" and "tintelingen" as its plural. "Tintelen" is a verb. "Tintelend" is an adjective.
} 
for instance, compared to "colors," with red, "a bit cliché," referring to a "glowing feeling" (Tara). David described sexual arousal as "a radiating warmth (...) like you're a kind of sun" and Lucas referred to energetic "waves" like when you "take XTC." Sandra was particularly creative with language and invented the term "flibble" ("fliebeltje"), a non-existent word that she then explained as "maybe like when something very soft touches you. That kind of feeling, but then on the inside." In these instances, informants translated the one embodied sensation, that of sex, into another embodied sensation, and thus still tried to capture what they experienced within a sensory register. The embodied experience was thereby made sense of by referring to another embodied experience.

Second, feelings were communicated by exemplifying their effect, in order to bypass the limitations of language (Willig 2007). Lucas, for instance, wanted to explain the pleasurable feeling he experiences when his partner touches the back of his upper leg. He suddenly inhaled very deeply and said: "That feeling." He later referred to this as a "breath-taking" sensation. Tara too indicated what she feels when she is aroused by her partner, and took a deep breath followed by a high pitched "hoooooh," to which she added: "You know, that kind of feeling." Tara then continued by saying, "It's that feeling of, 'Oh, how amazing,' you know like that. And also like, 'I now want to make out with you,' you know." While describing her embodied sensations, Tara sometimes placed her hand on her heart or on her hips, to indicate where the sensations are located, and also to seemingly connect to her embodied feelings. Similarly, Sophie made use of her body to visualize what she experiences when she really feels like sex:

[Two-second silence] I have the idea that my eyes are a bit wide-open, [Sophie widens her eyes] more open [Two-second silence]. And I feel some kriebel around my vagina. Yeah, kriebel is a bit of an odd description. [Three-second silence] Ehm, yeah, circulation of blood, ehm, tinteling, tinteling I think is better. Eh, yes, so a tinteling around my vagina.

Ehm, I sometimes feel my breasts are more present, and yeah, I stand more like this [Sophie arches her back and pushes her chest forward]. I'm more aware of my nipples.

The two ways of communicating explained here show that sensations are known to informants, yet beyond language. This is an important observation for sociological analysis. Data that are hard to capture are still, or even more, crucial for understanding the social. However, through joint-meaning making during the interviews, people managed to retrieve and "translate" their body-sensorial knowledge. Thus, they made this knowledge accessible to others-but also, simultaneously, to themselves. This is the classic insight of symbolic interactionism: people come to understand and know themselves only through their interactions with others.

\section{Discussion and Conclusion}

This article examined the process of verbalizing embodied sensations during interviews about sex, to gain insight into processes of joint sense-making in a domain where language is "found wanting." Sex, as an embodied, non-verbal practice proved to be a strategic domain to observe this process of "putting into words" embodied sensations. While sex is often seen as prediscursive or even pre-social, including by our informants, we show how doing, thinking, feeling, and talking about sex involves both embodied sensations and cultural knowledge. However, this is not a one-way street. Sex both mediates and shapes the social world in a "perceptual loop" between social meaning and body-sensorial knowledge. It does so largely without the intervention of language, the default human medium - and certainly the default medium of scientific enquiry. The difficulty of gathering knowledge about sex, as we were 
attempting to do in our interviews, arises not because people do not have this knowledge, or that it is pre-discursive or pre-social. Instead, it is difficult because this is body-sensorial knowledge, mediated by embodied interactions, and rarely put into words.

Our interviews aimed to analyze sexual practices and embodied sensations. During these interviews, we found that informants first had to become familiar with talking about sex and sexual sensations. The next step entailed identifying what is felt, which proved to be challenging for informants who made a distinction between experiencing sensations and being aware of what is experienced. Finally, informants verbalized their sensations, generally described as a "hard" and "difficult" task, in a variety of ways. We found that participants were creative and inventive in the ways they understood and communicated their bodily embodied sensations by: (1) using multi-interpretable sensory wordings; (2) drawing comparisons between different sexual sensations; and (3) referring to other sensory sensations, and exemplifying their effect (e.g. breathing, shrieking). In these steps, we gradually managed to uncover and make explicit the body-sensorial knowledge that informs the (sexual) actions and interactions of informants.

Concentrating on sexual practices and the productive character of body-sensorial knowledge implies a move away from dominant approaches to sexuality that take language as the focal point for analysis. Rather than taking speech as "data" for, or "end product" of the study of sex, we took the process of verbalizing embodied sensations itself as the focus of our study. Following Jackson and Scott, we used a combination of interactionism and practice theory to study sex as an embodied practice that unfolds in interactions. While informed by wider societal meaning systems, sex is also a site where social meaning is produced and recreated. This allowed us to conceptualize sex not as the product of discourse, as is common in the sociology of sex. Foucault-inspired studies see sex as the "acting out" of (heteronormative) discourses of modes of governmentality. While survey-based approaches clearly have a very different epistemological basis, they share a focus on bodily action as the enactment of "values" that are primarily conceptualized as words. Our approach, instead, highlights the productive character of body-sensorial knowledge. Essentially, we propose to investigate social life as processes of co-production between embodied sensation and cultural knowledge, visualized by the notion of a loop, a circle, to get away from more linear modes of scholarly inquiry.

While our analysis focuses on sex, we believe its relevance is much wider. First, we echo earlier calls to take seriously the embodiment of social life (Crossley 2001; Csordas 1997; Turner 2009; Wacquant 2004). While sex may seem to be an obvious case, it might serve to remind us that all human actions, and therefore all sociological studies, are about embodied actions and sensations. Second, we show that embodied actions and interactions can be "opened up" for reflection in the course of interviews - and thus be made accessible to sociological analysis. We offer a number of practical suggestions for doing this, and we also show that it is possible to "open up" such embodied sensations without losing sight of the layered and complex nature of these sensations. Finally, we present new conceptual tools to think about the relation between embodiment and knowledge. Processes of embodiment challenge the limitations of scholarly knowledge formation. Thus, they harbor possibilities to study the creation of social meanings. As speech is our main medium of studying embodied sensations, an analytical model to investigate the process of verbalizing embodied sensations will help future research to tap into this important aspect of human knowledge and experience: body-sensorial knowledge.

Acknowledgements The authors wish to thank all interviewees in this study for their time, openness, and enthusiasm. We also thank all members of the Culture Club at the University of Amsterdam for their useful advice. We greatly appreciate the feedback of the three anonymous reviewers and the editors, and are grateful for their insightful comments and suggestions. 
Funding This study was funded by The Netherlands Organisation for Scientific Research (Research Talent 2014).

\section{Compliance with ethical Standards}

Conflict of Interest The authors declare that they have no conflict of interest.

\section{Appendix}

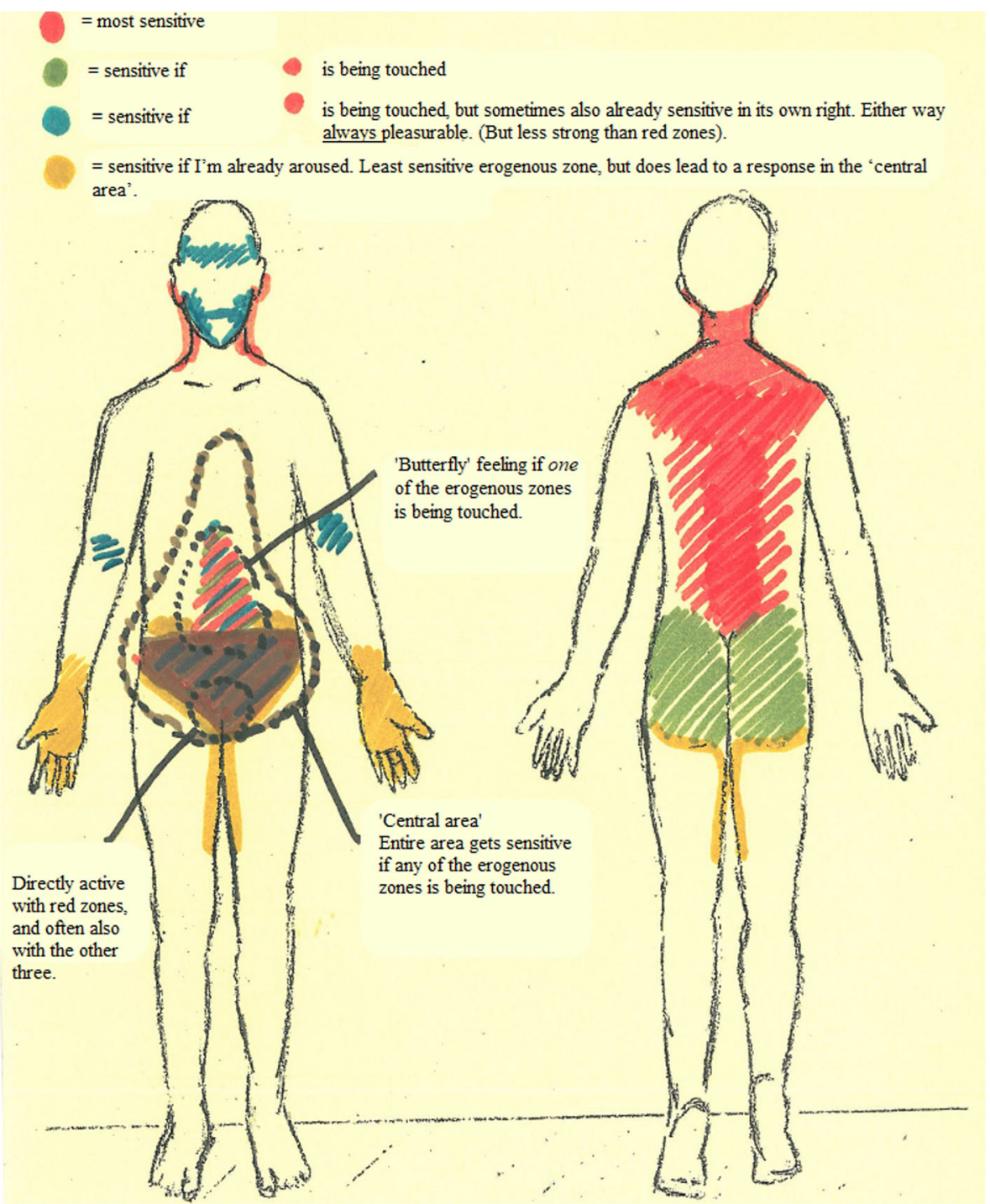

Fig. 1 Body Map, Simone. Note: Simone's handwriting was replaced by typed text (and translated into English) to protect confidentiality 
Open Access This article is distributed under the terms of the Creative Commons Attribution 4.0 International License (http://creativecommons.org/licenses/by/4.0/), which permits unrestricted use, distribution, and reproduction in any medium, provided you give appropriate credit to the original author(s) and the source, provide a link to the Creative Commons license, and indicate if changes were made.

\section{References}

Allen, Graham. 1980. A note on interviewing spouses together. Journal of Marriage and the Family 42 (1): $205-210$.

Allen-Collinson, Jacquelyn. 2009. Sporting embodiment: Sports studies and the (continuing) promise of phenomenology. Qualitative Research in Sport and Exercise 1 (3): 279-296.

Berger, Peter L., and Thomas Luckmann. 1991. The social construction of reality: A treatise in the sociology of knowledge. London: Penguin Books.

Bjørnholt, Margunn, and Gunhil R. Farstad. 2014. 'Am I rambling?' on the advantage of interviewing couples together. Qualitative Research 14(1):3-19.

Bordo, Susan. 1999. The male body. New York: Farrar, Straus and Giroux.

Bosman, Myra. 2012. Did you come? The (un)importance of the female orgasm during heterosex. Amsterdam: AMB publishers Diemen.

Cameron, Deborah, and Don Kulick. 2003. Language and sexuality. New York: Cambridge University Press.

Crossley, Nick. 2001. The phenomenological habitus and its construction. Theory and Society 30: 81-120.

Crossley, Nick. 2005. Sociology and the body. In The Sage handbook of sociology, eds. Craig Calhoun, Chris Rojek, and Bryan S. Turner, 442-456. London: Sage.

Csordas, Thomas J. 1990. Embodiment as a paradigm for anthropology. American Anthropological Association 18 (1): $5-47$.

Csordas, Thomas J. 1997. The sacred self: A cultural phenomenology of charismatic healing. Berkeley and Los Angeles: University of California Press.

De Jager, Adèle, Anna Tewson, Bryn Ludlow, and Katherine M. Boydell. 2016. Embodied ways of storying the self: A systematic review of body-mapping. Forum: Qualitative Social Research. https://doi.org/10.17169/fqs17.2.2526.

DeVault, Marjorie L. 1999. Liberating method: Feminism and social research. Philadelphia: Temple University Press.

Duncombe, Jean, and Dennis Marsden. 1996. Can we research the private sphere? Methodological and ethical problems in the study of the role of intimate emotion in personal relationships. In Gender relations in public and private: New research perspectives, eds. Lydia Morris and E. Stina Lyon, 141-155. London: Palgrave Macmillan.

Fine, Gary Alan. 2001. Enacting norms: Mushrooming and the culture of expectations and explanations. In Social norms, eds. Michael Hechter and Karl-Dieter Opp, 139-164. New York: Russell Sage Foundation.

Foucault, Michel. 1978. The history of sexuality. Volume 1: An Introduction. New York: Pantheon Books.

Frith, Hannah. 2013. Labouring on orgasms: Embodiment, efficiency, entitlement and obligations in heterosex. Culture, Health \& Sexuality 15 (4): 494-510.

Heaphy, Brian, and Anna Einarsdottir. 2012. Scripting civil partnerships: Interviewing couples together and apart. Qualitative Research 13 (1): 53-70.

Hekma, Gert. 2005. How libertine is the Netherlands? Exploring contemporary Dutch sexual cultures. In Regulating Sex: The Politics of Intimacy and Identity, eds. Elizabeth Bernstein and Laurie Schaffner, 209224. New York: Routledge.

Hite, Shere. 1976. The Hite report: A nationwide study on female sexuality. New York: Macmillan Publishing Co.

Hockey, Jenny, Angela Meah, and Victoria Robinson. 2007. Mundane heterosexualities: From theory to practices. Basingstoke: Palgrave.

Holstein, James A., and Jaber F. Gubrium. 1995. The active interview. Thousand Oaks: SAGE publications.

Jackson, Stevi. 2006. Gender, sexuality and heterosexuality: The complexity (and limits) of heteronormativity. Feminist Theory 7 (1): 105-121.

Jackson, Stevi, and Sue Scott. 2001. Embodying orgasm. Women \& Therapy 24 (1-2): 99-110.

Jackson, Stevi, and Sue Scott. 2004. Sexual antinomies in late modernity. Sexualities 7 (2): 233248.

Jackson, Stevi, and Sue Scott. 2007. Faking like a woman: Towards an interpretivist theorisation of sexual pleasure. Body \& Society 13 (2): 95-116.

Jackson, Stevi, and Sue Scott. 2010. Rehabilitating interactionism for a feminist sociology of sexuality. Sociology 44 (5): 811-826. 
Jackson, Stevi, and Sue Scott. 2017. Towards a practice theoretic analysis of sexuality. In Sexualities research: Critical interjections, diverse methodologies and practical applications, eds. Andrew King, Ana Cristina Santos, and Isabel Crowhurst, 70-82. London: Routledge.

Kinsey, Alfred C., Wardell B. Pomeroy, and Clyde E. Martin. 1948. Sexual behavior in the human male. Philadelphia: W.B. Saunders Company.

Kinsey, Alfred C., Wardell B. Pomeroy, Clyde E. Martin and Paul H. Gebhard. 1953. Sexual behavior in the human female. Philadelphia and London: W.B. Saunders Company.

Laumann, Edward O., John H. Gagnon, Robert T. Michael, and Stuart Michaels. 1994. The social organization of sexuality: Sexual practices in the United States. Chicago: University of Chicago Press.

Lindemann, Gesa. 1997. The body of gender difference. In Embodied practices: Feminist perspectives on the body, ed. Kathy Davis, 73-92. London: Sage.

MacGregor, Hayley N. 2009. Mapping the body: Tracing the personal and the political dimensions of HIV/AIDS in Khayelitsha, South Africa. Anthropology \& Medicine 16 (1): 85-95.

Merleau-Ponty, Maurice. 1962. Phenomenology of perception. London: Routledge \& Kegan Paul.

Mepschen, Paul, Jan-Willem Duyvendak, and Evelien H. Tonkens. 2010. Sexual politics, orientalism, and multicultural citizenship in the Netherlands. Sociology 44 (5): 963-979.

Milani, Tomasso M. 2017. Language and sexuality. In The Oxford handbook of language and society, eds. Ofelia García, Nelson Flores, and Massimiliano Spotti, 403-422. New York: Oxford University Press.

Plummer, Ken. 2003. Queer, bodies and postmodern sexualities: A note on revisiting the "sexual" in symbolic interactionism. Qualitative Sociology 26 (4): 515-530.

Pugh, Allison J. 2013. What good are interviews for thinking about culture? Demystifying interpretive analysis. American Journal of Cultural Sociology 1 (1): 42-68.

Reckwitz, Andreas. 2012. Affective spaces: A praxeological outlook. Rethinking History 16 (2): 241-258.

Rubin, Gayle S. 1984. Thinking sex: Notes for a radical theory of the politics of sexuality. In Pleasure and danger: Exploring female sexuality, eds. Carol Vance, 267-319. London: Pandora.

Schalet, Amy. 2011. Not under my roof: Parents, teens, and the culture of sex. Chicago: Chicago University Press.

Schutz, Alfred. 1970. Alfred Schutz on phenomenology and social relations: Selected writings, ed. Helmut R. Wagner. Chicago: The University of Chicago Press.

Spronk, Rachel. 2014. Sexuality and subjectivity: Erotic practices and the question of bodily sensations. Social Anthropology/Anthropologie Sociale 22 (1): 3-21.

Stets, Jan, and Jonathan H. Turner. 2014. Handbook of the sociology of emotions. Vol. 2. Berlin: Springer.

Turner, Jonathan H. 2009. The sociology of emotions: Basic theoretical arguments. Emotion Review 1 (4): 340-354.

Van Hooff, Jenny. 2013. Modern couples? Continuity and change in heterosexual relationships. Farnham: Ashgate Publishing Limited.

Wacquant, Loïc. 2004. Body \& Soul: Notebooks of an apprentice boxer. New York: Oxford University Press.

Warde, Alan. 2014. After taste: Culture, consumption, and theories of practice. Journal of Consumer Culture 14(3):279-303.

Warde, Alan. 2016. The practice of eating. Cambridge: Polity Press.

Weber, Max. 1978. Economy and society: An outline of interpretive sociology, eds. Guenther Roth and Claus Wittich, Vol. 1. Berkeley: University of California Press.

Wilkinson, Ian. 2001. Thinking with suffering. Cultural Values 5 (4): 421-444.

Willig, Carla. 2007. Reflections on the use of a phenomenological method. Qualitative Research in Psychology 4 (3): 209-225.

Zussman, Robert. 2004. People in places. Qualitative Sociology 27 (4): 351-363.

Publisher's Note Springer Nature remains neutral with regard to jurisdictional claims in published maps and institutional affiliations.

Myra Bosman is a PhD Candidate in Anthropology and Sociology at the University of Amsterdam. Her doctoral research focusses on heteronormativity and heterosexual sex practices in The Netherlands. It is based on interviews with man-woman couples, the analysis of a large online forum, and the study of a reality TV series on sex education for adults.

Rachel Spronk is Associate Professor at the Anthropology department of the University of Amsterdam. She is the author of Ambiguous Pleasures: Sexuality and Middle Class Self-Perceptions in Nairobi (New York/Oxford, 2012) and has written various articles on sex, gender, love, and cosmopolitanism. Her projects (Ghana, Kenya, Netherlands) evidence a concern with the historical trajectories that have shaped the present, the production of knowledge about gender and sexuality, and ultimately, how the lived experiences of people inform our theoretical models. 